\title{
Fabrication of holographic gratings using a moving point source
}

\author{
Daniel Post, James McKelvie, Meirong Tu, and Fu-long Dai
}

\begin{abstract}
In the manufacture of holographic gratings using coherent light there is an associated problem of optical noise, the conventional remedies for which involve various restrictions. In this work a moving point source is adopted and analysis indicates that if the path is a circle several of these restrictions are removed. The scheme is implemented and the results are highly satisfactory with high-efficiency gratings being produced at frequencies up to 2400 lines $/ \mathrm{mm}$, in sizes up to $65 \mathrm{~mm}$ square. Significant operational advantages also accrue in relation to alignment procedures and light utilization.
\end{abstract}

\section{Introduction}

In the production of gratings by the interference of two coherent beams of light, the presence of optical noise caused by dust and local imperfections of the optical elements causes a reduction in the diffraction efficiency. This noise also produces what may in some applications be a more serious effect, namely, a background scattering which degrades the quality of the information carried by the major diffracted beams. For example, in moire interferometry, ${ }^{1}$ this degradation is manifested as the intensity of the speckle in a fringe pattern. Attempts to reduce the noise by the use of a distributed source to limit the spatial coherence, ${ }^{2}$ or a broad spectrum to limit the temporal coherence, ${ }^{2,3}$ impose certain penalties: the useful size of the product grating may be restricted ${ }^{2}$; the fringe contrast may be variable ${ }^{2}$; the demands on interferometer alignment may be severe. ${ }^{3}$

Swanson ${ }^{2}$ gives first- and second-order analyses of the behavior of the Mach-Zehnder type of interferometer used for the production of gratings with a monochromatic source that is broad and spatially incoherent. One quoted method of creating such a source is to place a rotating piece of diffuse glass close to a coherent source.

Those analyses explicitly assume that any two points in such a source are completely incoherent with

Daniel Post is with Virginia Polytechnic Institute \& State University, Department of Engineering Science \& Mechanics, Blacksburg, Virginia 24061-0219, James McKelvie is with Strathclyde University, Scotland, Meirong Tu is with Chinese Academy of Sciences, and Fu-long Dai is with Tsinghua University, China.

Received 3 October 1988.

0003-6935/89/163494-04\$02.00/0.

(c) 1989 Optical Society of America. each other. However, this assumption may not be entirely valid. In the present work, a moving point source is adopted, which fulfills the incoherency condition.

In his insightful analyses, ${ }^{2}$ Swanson shows that the holographic grating could be unrestricted in size and perfectly uniform if a diffuse circular line source (as opposed to a disk area source) is used. Equally then, a point source moving in a circle should produce optimal results. However, Swanson's analysis involves approximations to the second order and considers (although this is not explicitly stated) the case of relatively coarse gratings. The following geometric argument is exact and applies to gratings of any frequency.

\section{Theoretical Background}

As in Ref. 2, the Mach-Zehnder type of interferometer (Fig. 1) is visualized as producing, from one planar source, a system of two identical virtual planar sources $S$ and $S^{\prime}$, as illustrated in Fig. 2. The sources are not mirror images, which is indicated by arrows to corresponding points $A$ and $A^{\prime}$. The system is taken to be aligned such that the planes containing the sources are disposed symmetrically with respect to the $y$ - $z$ plane, as illustrated in Fig. 2. These source planes are normal to $O C$ and $O C^{\prime}$, where $C$ and $C^{\prime}$ are corresponding points in the source planes, each at a distance $x_{c}$ from the $z$ axis. The $x-y$ plane is the recording plane of Fig. 1. We may now say

$$
\sin \alpha_{c}=\frac{x_{c}}{d_{c}} ; \quad \cos \psi=\frac{d_{c}}{P} ; \quad \sin \alpha=\frac{L}{R} .
$$

Line $A A^{\prime}$ (of length $2 L$ ) is parallel to the $x-z$ plane. Considering the projections in the $x-z$ plane, close inspection reveals

$$
\sin \alpha_{c}=\frac{L}{P}
$$




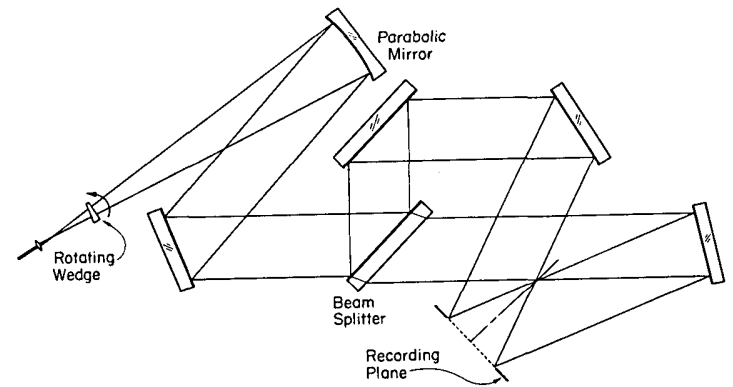

Fig. 1. Mach-Zehnder interferometer arrangement for grating manufacture, showing the position of a rotating wedge.

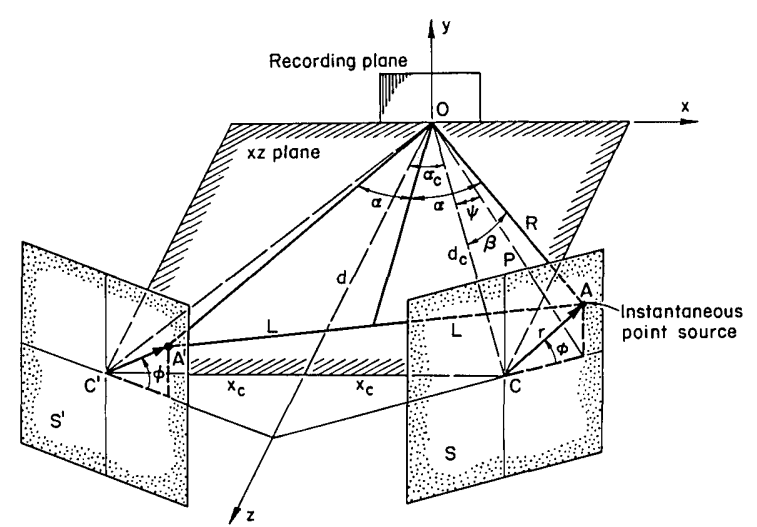

Fig. 2. Geometry of twin virtual sources in symmetrically disposed planes.

The two point sources will produce a Young's fringe system, with a frequency in a small volume surrounding $O$ given by

$$
f=\frac{2 \sin \alpha}{\lambda},
$$

where the frequency is measured along a line lying at angle $\psi$ to $O X$ in the $x-z$ plane. Along $O X$, therefore, the fringe frequency is

$$
f^{\prime}=f \cos \psi
$$

Combining Eqs. (1), (2), and (3) yields

$$
f^{\prime}=\frac{2 x_{c}}{\lambda R}
$$

Therefore, the frequency $f^{\prime}$ does not vary so long as $R$ is constant. This condition is achieved when the source points move in circles centered on $C$ and $C^{\prime}$, such that radius $r$ and angle $\beta$ are constants (Fig. 2). Under this circumstance, $\alpha$ and $\psi$ both vary with $\phi$, but they vary harmoniously such that $f^{\prime}$ remains constant.

It is perhaps noteworthy that Eq. (4) does not give exactly the same frequency for the circular source as Ref. 2. Consideration of Fig. 2, with the instantaneous sources directly above $C$ and $C^{\prime}$, shows that Eq. (4) is correct.

\section{A. Size Restrictions}

Of course, the grating frequency varies as we move away from $O$, but if the source goes in effect to infinity

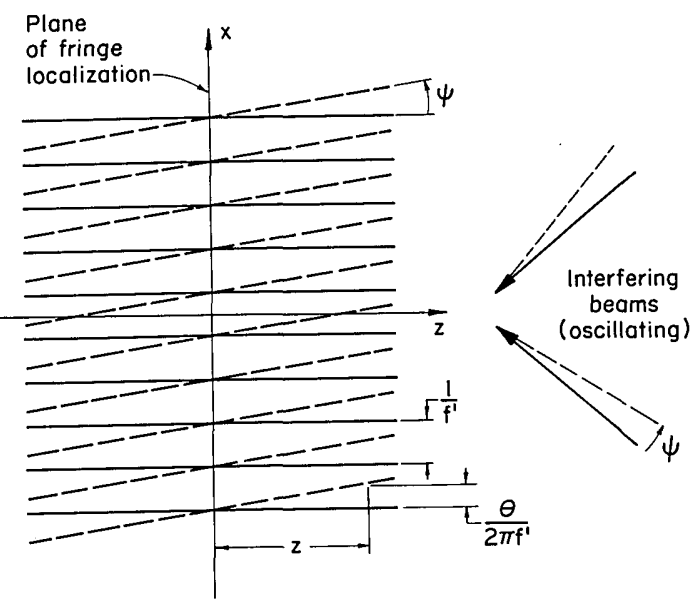

Fig. 3. $z$-direction dependence of grating phase as interfering beams oscillate.

(by the use of a suitable collimator) the grating frequency will be constant within the dimensions of the collimated beams and will vary only with any departure from wavefront flatness. Moreover, it is clear from the geometry that the path lengths from the two sources to the origin do not vary, so that the phase of the grating constructed from different points of the circles is always the same (with an intensity maximum at $O$ in a perfectly symmetrical arrangement).

Thus, high contrast gratings over large dimensions $x$ and $y$ are predicted.

\section{B. z-Direction Localization}

The grating of frequency $f^{\prime}$ in the $x-y$ plane is formed, as shown above, by the intersection of a fringe system of frequency $f$ whose system axis lies at the angle $\psi$ to the recording plane. This angle oscillates between $\pm \psi_{\max }= \pm \beta$ as the source moves in a circle of radius $r$. For any plane other than $x y$, therefore, the phase of the grating is continually changing, and a limit is thus placed on the depth in the $z$-direction within which the summation of exposure over one revolution of $\phi$ can lead to a satisfactory grating.

Considering Fig. 3, the plane where the maximum deviation of the grating phase angle is $\pm \theta \mathrm{rad}$ is located at

$$
z= \pm \frac{\theta}{2 \pi f^{\prime} \tan \psi_{\max }}= \pm \frac{\theta}{2 \pi f^{\prime} \tan \beta} .
$$

If a deviation $\theta=\pi / 5$ is accepted as the limit to yield satisfactory gratings, the zone of acceptable fringe localization, or zone of tolerance $z_{t}$, is given by

$$
z_{t}= \pm \frac{1}{10 f^{\prime} \tan \beta} .
$$

The angle subtended at $O$ by the source circle is $2 \beta$ when using a collimator of focal length $F$, where $\tan \beta=$ $r / F$. Accordingly,

$$
z_{t}= \pm \frac{F}{10 r f^{\prime}}
$$




\section{Experimental Work}

\section{A. Apparatus}

An interferometer was assembled as shown in Fig. 1, with an apparent circular movement of the source being effected by the rotation of a glass wedge; typically, a $1^{\circ}$ or $2^{\circ}$ wedge was used. The position of the wedge was variable in the $0-100-\mathrm{mm}$ range from the source, providing the $0-2-\mathrm{mm}$ range (approx.) for the radius of the circular path of the point source. The collimator was $150-\mathrm{mm}$ diameter and $1220-\mathrm{mm}$ focal length. One Watt in the 413-nm wavelength was available from a krypton-ion laser.

\section{B. Alignment}

As the analysis indicates, it was necessary to bring the interferometer into the described condition of symmetry. This was done in successive steps. First, with an initial setup that used an unexpanded beam, particular attention was paid to arranging a constant beam height from the table. Next, the interference pattern in the recording plane-this pattern is called a virtual grating-was adjusted in frequency by a scheme illustrated in Fig. 4. A transmission-type diffraction grating of half the desired frequency was installed in the recording plane. Using a broad collimated beam, interference of the +1 and -1 diffraction orders, one from each beam, was observed on a screen. The interference fringes resulting from interaction of the virtual grating and the real grating are called moire fringes. The interferometer mirrors was adjusted to obtain the infinite fringe condition (zero moire fringe gradient) across the screen. This assured a virtual grating frequency in the recording plane of twice the frequency of the transmission grating.

To achieve a first approximation of system symmetry, a positive lens of long focal length was inserted upstream of the beam splitter, to bring the beam to a minimum cross section in the recording plane. Two bright dots appeared on the recording plane. The dots were merged into one. Their horizontal separation (parallel to the table) was controlled by translating the recording plane toward or away from the mirrors; their vertical separation was controlled by fine adjustment of one interferometer mirror.

The lens was removed, the source was made to rotate, and the infinite fringe condition was tuned and viewed on the screen (Fig. 4). The transmission grating was rotated (slightly) in its plane to produce a few parallel fringes on the screen. With the rotating source, the fringes moved back and forth in synchronism with the wedge rotation. By fine adjustment (translation and rotation) of the plane of the transmission grating and fine vertical adjustment of one beam coming from the interferometer, the system was tuned to a fractional fringe movement, as judged by eye. Provisions for tilting and translating the recording plane fixture were present to effect these adjustments. The adjustments for fringe movement were usually done with the maximum radius of the source circle and the radius was subsequently reduced for producing

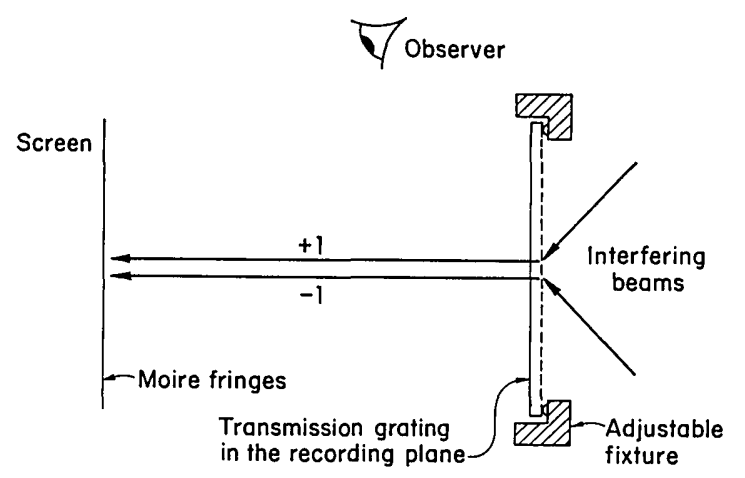

Fig. 4. Arrangement for observing moire fringes while fine tuning the interferometer alignment.

gratings. With some patience the system could be tuned to give essentially no fringe movement even with a source path radius of $2 \mathrm{~mm}$. This alignment procedure provided the required system symmetry.

\section{Results}

Numerous crossed-line gratings of frequency $1200 \times$ 1200 lines/mm were produced in Shipley Microposit 1400 resist, on glass, over a field $65 \mathrm{~mm}$ square. With 1.0-W laser power, an exposure time of $12 \mathrm{~s}$ was used for each direction. The efficiency was such that, after aluminizing, over $90 \%$ of the light was directed into the four first-order diffractions for $\lambda=632.8 \mathrm{~nm}$. Many gratings of 2400 -lines/mm frequency, both linear and crossed, were also produced. It proved possible, by adjustment of the exposure time, to tailor broadly the ratio of the energies in the diffracted and zero orders.

A dramatic reduction in the optical noise was achieved with a source circle radius of $1 \mathrm{~mm}$. One criterion for the noise level associated with a grating is the maximum fringe frequency that can be resolved when two diffracted orders are brought to interference. In this work, using a 1200-lines/m grating, fringe frequencies of 600 lines $/ \mathrm{mm}$ were observed in a moire interferometer arrangement. This was near the limit of resolution of the imaging lens used, yet the fringes were of high contrast, dominating the noise.

\section{Practical Considerations}

The moving point source arrangement provides two important advantages when compared to a broad monochromatic source arrangement. They are improved utilization of the available light and relatively easy system alignment.

In the case of a diffuse source there are an infinite set of fringe systems simultaneously present in the region by the recording plane, and these combine to give good contrast fringes only in a narrow volume of space. This characteristic makes the determination of the position for the recording plane a laborious business. ${ }^{3}$ The property of the moving source arrangement, whereby at any time there is but one fringe system, allows a very straightforward tuning process. The fringes produced with the transmission diffraction grating (Fig. 4) can be observed in the whole space 
behind the grating, and the only remaining task is to make their movement adequately small. This is an easy judgment to make, compared to a critical assessment of fringe contrast across the field. As the fringe frequency increases, this aspect assumes increasing importance, since the localization zone becomes narrower.

The light utilization is many times that of the diffuse broad source which tends to radiate a good portion of the energy in wasteful directions. Moreover, the useful feature of the broad source, whereby the Gaussian profile of beam intensity is suppressed, has an equivalent mechanism in the case of the moving source. Such a feature is of significance when uniformity of diffraction efficiency across a broad field is important.

Practical assessment of the depth of localization with a 1-mm source radius indicated an acceptable zone of a few tenths of millimeters with $f^{\prime}=1200$ lines/ $\mathrm{mm}$. This is consistent with Eq. (5).

The effect of the rotating source manifests itself very obviously as a rotation of the noise, which can be seen to move in circles that are larger for noise sources further from the recording plane. It seems desirable, therefore, to keep the two final mirrors as far from the recording plane as is practicable. The fact that these noise circles have various radii, with the additional observation that the rotations are not concentric, appear to be helpful in the averaging out of the noise.

\section{v. Conclusions}

In the interferometric production of gratings, the use of a point source moving in a circular path is effective as a means of reducing coherent noise. Two important advantages are improved light utilization and relative ease of alignment. The test for final adjustment is objective, based on fringe movement instead of fringe contrast. The arrangement is effective for high frequencies; excellent crossed line gratings of 1200 and 2400 lines $/ \mathrm{mm}$ were made in photoresist.

Support for this work from the Office of Naval Research under contract N00014-86-K-255, from the National Center for Composite Materials Research subcontract under ONR contract N00014-86-K0799, from the Chinese National Scientific Fund Committee, and from the U.S. National Academy of Sciences under the exchange program of the Committee on Scholarly Communication with the People's Republic of China, is gratefully acknowledged.

\section{References}

1. D. Post, "Moire Interferometry," in Handbook of Experimental Mechanics, A. S. Kobayashi, Ed. (Prentice-Hall, Englewood Cliffs, NJ, 1987), chap. 7.

2. G. J. Swanson, "Broad-Source Fringes in Grating and Conventional Interferometers," J. Opt. Soc. Am. A 1, 1147-1153 (1984).

3. D. Post, K. Patorski and P. Ning, "Compact Grating Interferometer for Producing Photoresist Gratings with Incoherent Light," Appl. Opt. 26, 1100-1105 (1987).
Of Optics continued from page 3396

duration than those being transmitted now. The shorter each signal was, the more data could be relayed in a single second. At Spectra-Physics, Inc., James Kafka, a researcher, and Thomas Baer, a senior research fellow, have built devices that produce soliton pulses as short as four-trillionths of a second. That is about 250 times shorter than pulses transmitted over the most advanced fiber networks today. They believe pulses as short as a ten-trillionth of a second should soon be possible. In theory, if such pulses could be incorporated into a communications system, the researchers said it would be possible to transmit the entire contents of the Library of Congress in about 100 seconds. "Part of the appeal of solitons is that there is something magical to their qualities," Kafka said. "It's almost a something-for-nothing kind of deal."

A number of obstacles remain, however. For one thing, even soliton light pulses lose energy as they travel down the fiber, and that eventually reduces their stability. To prevent that, laser diodes to replenish lost light must be installed at regular intervals in the optical fiber. That is still much simpler than an electronic repeater. The problem, however, is that commercially available laser diodes are not quite strong enough to propel solitons for long distances,
Mollenauer said. The problem becomes more difficult as speeds increase. As light pulses become shorter, they require greater energy to become solitons. As a result, speeds on a par with the Spectra-Physics laser device are not considered likely soon. Indeed, some fiber optics researchers point out that other emerging fiber technologies may make ordinary light pulses more practical than solitons. A new generation of optical amplifiers, for example, could replace the sluggish electronic repeaters in use today and make ordinary light signals much faster.

Even if all of these issues are resolved, it will be necesary to build processing equipment that is fast enough to code and decode the light pulses on either end of a communication. Even long-distance telephone carriers cannot take full advantage of today's transmission speeds, and private communications customers have even less capability to process information at such speeds. As a result, some experts suggest caution in making predictions about the future. "A long time ago, people were pointing to picture phones as the real reason to push fiber optics," said Richard Claus, director of the Fiber and Electro-Optics Research Center at Virginia Polytechnic Institute in Blacksburg, VA. "Well, fiber optics are here, but picture phones still aren't" in widespread use. 